\title{
Desempenho e avaliação econômica da alimentação de cordeiros confinados com dietas contendo diferentes relações volumoso:concentrado
}

\author{
Performance and economic evaluation of the feeding of feedlot lambs with diets with different \\ roughage:concentrate ratios
}

\author{
Sérgio Carvalho' ${ }^{\mathrm{I}}$ Mariana Augusta Brochier ${ }^{\mathrm{I}}$ Josane Pivato $^{\mathrm{I}}$ André Vergueiro ${ }^{\mathrm{II}}$ \\ Regina Cánovas Teixeira ${ }^{\mathrm{III}}$ Roberto Kieling ${ }^{\mathrm{III}}$
}

\section{RESUMO}

Este estudo objetivou avaliar o efeito do uso de diferentes relações volumoso:concentrado sobre o desempenho, as características de carcaça e o conteúdo gastrintestinal de cordeiros, bem como realizar uma avaliação econômica da alimentação de cordeiros confinados na fase de terminação. Foram utilizados 25 cordeiros, machos não-castrados, da raça Texel, desmamados com a idade média de 60 dias, $e$ distribuídos aleatoriamente em cinco tratamentos, compostos por diferentes relações volumoso:concentrado, sendo: 30:70; 40:60; 50:50; 60:40 e 70:30, em base da matéria seca (MS). $O$ volumoso utilizado foi o feno de Tifton-85 e o alimento concentrado era constituído por ração comercial, farelo de soja, calcário calcítico e fosfato bicálcico, em diferentes proporções. O período experimental teve duração de 80 dias, quando os animais foram abatidos. $O$ aumento do teor de volumoso e a diminuição no teor de concentrado nas dietas promoveu redução linear $(P \leq 0,01)$ no ganho de peso diário e peso vivo ao abate. $O$ consumo de matéria seca e a conversão alimentar foram influenciados de forma quadrática pelo aumento da relação volumoso:concentrado. Verificou-se redução linear $(P \leq 0,01)$ no peso de carcaça quente, rendimento de carcaça quente e nos pesos de quarto (perna), paleta, costilhar, pescoço e de vísceras verdes vazias, com o aumento do teor de volumoso e a diminuição no teor de concentrado nas dietas. Ocorreu crescimento linear nas proporções de vísceras verdes cheias $(P \leq 0,05)$ e de conteúdo gastrintestinal $(P \leq 0,01)$ com a elevação da proporção de volumoso e com a redução na proporção de concentrado nas dietas. A lucratividade em relação à venda dos cordeiros vivos não diferiu entre os tratamentos, apresentando um valor médio de $R \$ 57,00$ por cordeiro. Houve redução linear $(P \leq 0,05)$ na lucratividade em relação à venda das carcaças dos cordeiros com o aumento da proporção de volumoso e com a diminuição na proporção de concentrado nas dietas.
Palavras-chave: componentes do peso vivo, confinamento, ovinos, nutrição.

\section{ABSTRACT}

This study was aimed at evaluating the effect of the use of different roughage:concentrate ratios on the performance, carcass characteristics and gastrintestinal content, and realizeing an economic evaluation of the feeding of feedlot lambs in termination phase. Twenty-five male lambs, of the Texel breed, non castrate, weaned with 60 days of age, were used. They were randomly distributed in five treatments, composed by different roughage:concentrate ratios, being: 30:70; 40:60; 50:50; 60:40 and 70:30, on dry matter basis (DM). The roughage used was the Tifton-85 hay and the concentrate was composed by commercial ration, soybean meal, limestone and dicalcium phosphate, in different proportions. The experimental period was 80 days long, when the animals were slaughtered. The increase of the roughage and the decrease of the concentrate on the diets promoted a linear reduction $(P \leq 0.01)$ on the daily weight gain and slaughter live weight. The consume of dry matter and food conversion were influenced in a quadratic way by the increased of the relation roughage:concentrate. The increased of the roughage and the decreased of the concentrate on the diets promoted a linear decreased $(P \leq 0.01)$ on the hot carcass weight, hot carcass yield and weight of hindquarter, neck, rib, shoulder and of empty green viscera. It was verified a linear increase of the proportions of full green viscera $(P \leq 0.05)$ and the gastrintestinal content $(P \leq 0.01)$ with the increase of the proportion of roughage and decreased of the concentrate in the diets. The sales profitability of the live lambs was not different between the treatments, showing an average value of $R \$ 57.00$ per lamb. There was a linear decrease $(P \leq 0.05)$ in the profitability in the sale of lamb's carcass with the increased of roughage and decrease of concentrate in the diets.

'Programa de Pós-graduação em Gestão Tecnológica: qualidade ambiental. Centro Universitário Feevale, RS 239, 2755, 93352000, Novo Hamburgo, RS, Brasil. E-mail: sergiocarvalho@feevale.br. Autor para correspondência.

"Empresa BÜNGE Alimentos S.A., Porto Alegre, RS, Brasil.

II'Curso de Engenharia de Produção - Habilitação Agroindustrial, Centro Universitário Feevale, Novo Hamburgo, RS, Brasil. 
Key words: live weight components, confinement, sheep, nutrition.

\section{INTRODUÇÃO}

Com o crescimento da população mundial, existe a necessidade de aumentar a eficiência dos sistemas de produção de alimentos, nos quais a produção de proteína de origem animal assume grande importância. Neste sentido, CARVALHO (2005) citou que os ovinos desempenham um papel produtivo importante em várias partes do mundo, transformando plantas forrageiras em proteína alimentar de elevado valor biológico, contribuindo para combater a fome e assumindo um papel de extrema relevância frente ao crescimento das populações.

Contudo, em algumas regiões do Brasil, há o predomínio de pequenas e médias propriedades rurais, nas quais há limitação de área para produção de carne ovina sobre pastejo. Além disso, nestas condições, normalmente ocorre uma alta concentração de carga parasitária nas pastagens, tornando a infestação por verminose um grave problema, que tem levado a sérios prejuízos econômicos. O confinamento de cordeiros é uma alternativa interessante, que poderá contribuir com o incremento de renda de propriedades rurais que tenham limitação de área de pastagem disponibilizada para produção animal.

O confinamento de cordeiros apresenta uma série de benefícios, como menor mortalidade dos animais devido à menor incidência de verminoses (SIQUEIRA et al., 1993) e maior controle da parte nutricional. Além disso, o confinamento de cordeiros agiliza o retorno do capital aplicado, permite a produção de carne de qualidade durante todo o ano, permite padronização de carcaças, reduz a idade de abate dos cordeiros e disponibiliza a forragem das pastagens para as demais categorias do rebanho. No entanto, as maiores desvantagens se encontram nos altos custos de produção, principalmente, relacionados à alimentação.

De acordo com BENDAHAN (2006), a decisão da utilização do confinamento de cordeiros é puramente econômica, na qual fatores como velocidade de acabamento, conversão alimentar, qualidade dos animais disponíveis, preço e qualidade da alimentação e mercado demandador de carne de qualidade devem ser levados em conta sistematicamente, para que o produtor obtenha ganho econômico na atividade.

O confinamento de cordeiros recémdesmamados, com uso de altos níveis de concentrado, é uma prática que vem sendo cada vez mais utilizada, objetivando-se a redução da idade de abate e a obtenção de carcaças de qualidade. Contudo, de acordo com GASTALDI \& SOBRINHO (1998), a maximização do uso de concentrados no confinamento acarreta, geralmente, aumento do custo de produção e maior possibilidade de ocorrência de distúrbios fisiológicos nos animais. Entretanto, permite rações com maior concentração de nutrientes, o que pode ser interessante quando se dispõe de animais com alto potencial para ganho de peso.

Por outro lado, MERTENS (1992) cita que o uso de dietas com altas concentrações de volumoso pode levar a uma regulação física do consumo de nutrientes devido ao efeito físico provocado pelo teor de fibra em detergente neutro (FDN) e, desta maneira, influenciar de maneira negativa sobre o desempenho animal. Portanto, estudos que avaliem a eficiência do uso de diferentes relações volumoso:concentrado poderão contribuir com a busca da eficiência produtiva e econômica da terminação de cordeiros em confinamento.

Neste sentido, o presente estudo objetivou avaliar o efeito do uso de diferentes relações volumoso:concentrado sobre o desempenho, as características de carcaça e o conteúdo gastrintestinal de cordeiros terminados em confinamento, bem como realizar uma avaliação econômica da alimentação utilizada.

\section{MATERIAL E MÉTODOS}

O experimento foi conduzido nas instalações de ovinocultura de uma propriedade rural localizada no município de Capela de Santana, RS, no período de 02 de outubro a 21 de dezembro de 2004.

Foram utilizados 25 cordeiros, machos nãocastrados, da raça Texel, desmamados com 60 dias de idade, e distribuídos aleatoriamente em cinco tratamentos, em sistema de confinamento, em baias cobertas, com piso ripado e suspensas do solo. Os tratamentos foram constituídos por diferentes relações volumoso:concentrado, sendo: 30:70; 40:60; 50:50; 60:40 e 70:30, em base da matéria seca (MS). O volumoso utilizado foi o feno de Tifton-85, e o alimento concentrado era constituído por ração comercial, farelo de soja, calcário calcítico e fosfato bicálcico. As dietas eram isoprotéicas, baseadas no teor de proteína bruta da dieta composta exclusivamente por feno de Tifton85 e ração comercial, na proporção 70:30, na MS. A proporção dos ingredientes utilizados e a composição química das dietas experimentais são apresentadas na tabela 1 .

Ciência Rural, v.37, n.5, set-out, 2007. 
Tabela 1 - Proporção dos ingredientes e composição química das dietas experimentais.

\begin{tabular}{|c|c|c|c|c|c|}
\hline & \multicolumn{5}{|c|}{ Relação volumoso:concentrado } \\
\hline & $30: 70$ & 40:60 & $50: 50$ & $60: 40$ & $70: 30$ \\
\hline \multicolumn{6}{|c|}{ Proporção dos ingredientes (\% MS) } \\
\hline Feno de Tifton-85 & 30 & 40 & 50 & 60 & 70 \\
\hline Ração comercial & 70 & 54,79 & 39,67 & 24,48 & 9,38 \\
\hline Farelo de soja & ----- & 4,43 & 8,82 & 13,29 & 17,63 \\
\hline Calcário calcítico & ---- & 0,7 & 1,36 & 2,10 & 2,77 \\
\hline Fosfato bicálcico & ----- & 0,08 & 0,15 & 0,18 & 0,23 \\
\hline \multicolumn{6}{|c|}{ Composição química (\% MS) } \\
\hline MS & 86,4 & 86,35 & 86,28 & 86,23 & 86,17 \\
\hline $\mathrm{PB}$ & 18,3 & 18,3 & 18,3 & 18,3 & 18,3 \\
\hline FDN & 29,7 & 36,66 & 43,63 & 50,59 & 57,56 \\
\hline NDT & 64,3 & 62,61 & 60,94 & 59,26 & 57,60 \\
\hline $\mathrm{Ca}$ & 1,53 & 1,53 & 1,53 & 1,53 & 1,53 \\
\hline $\mathrm{P}$ & 0,34 & 0,34 & 0,34 & 0,34 & 0,34 \\
\hline
\end{tabular}

$\mathrm{O}$ alimento foi fornecido, ad libitum, duas vezes ao dia. A quantidade oferecida foi ajustada em função da sobra observada diariamente, sendo que esta deveria ser de $10 \%$ da quantidade oferecida no dia anterior, de modo a garantir o consumo voluntário máximo dos animais. Neste sentido, foram realizadas pesagens diárias das sobras e do alimento oferecido. Foram obtidas amostras compostas do feno oferecido, dos concentrados e das sobras, as quais foram congeladas em freezer e posteriormente analisadas na Central Analítica do Centro Universitário Feevale, conforme metodologias descritas por SILVA(1990). Em todos os tratamentos encontrava-se água e sal comum à vontade.

O período experimental foi precedido por um período pré-experimental de 14 dias, que serviu para adaptar os animais ao desmame, às instalações, à alimentação e ao manejo. Os abates foram precedidos de um jejum de sólidos e líquidos por 14 horas, tendo sido realizados após um período experimental de 80 dias. Após cada abate, procedeu-se à pesagem do trato gastrintestinal cheio e, em seguida, realizou-se esvaziamento e minuciosa lavagem do mesmo. Após escorrimento da água, o trato gastrintestinal foi pesado vazio. Por diferença, obteve-se o peso do conteúdo gastrintestinal. Em seguida, as carcaças dos animais foram pesadas e, logo depois, separadas regionalmente nos seguintes cortes comerciais: Pescoço: porção compreendida entre a secção atlanto-ocipital e um corte oblíquo que passa entre a sétima vértebra cervical e a primeira dorsal, em direção à ponta do esterno, terminando na borda inferior do pescoço; Paleta: membro anterior da carcaça, incluindo a musculatura da escápula e, na parte distal, a secção é feita ao nível da porção média dos ossos do carpo; Costilhar: parte da carcaça seccionada entre a última vértebra cervical e a primeira torácica e entre a última lombar e a primeira sacra; Perna ou quarto: membro posterior da carcaça, seccionado ao nível da articulação da última vértebra lombar e da primeira sacra e ao nível da porção média do tarso.

Após a separação regional, os diferentes cortes comerciais foram pesados e sua porcentagem calculada em relação ao peso da carcaça. A separação regional foi realizada segundo OSÓRIO et al. (1998).

Para se efetuar a análise econômica da alimentação oferecida no experimento, foram considerados os preços de mercado obtidos para os ingredientes das rações, para a carcaça e o peso vivo de cordeiros. De posse do custo de cada ração e do consumo de matéria seca das mesmas, foi calculado o resultado econômico proporcionado por ração. Utilizouse um valor de $\mathrm{R} \$ 3,00 \mathrm{~kg}^{-1}$ de peso vivo dos cordeiros, $\mathrm{R} \$ 9,50 \mathrm{~kg}^{-1}$ de carcaça, $\mathrm{R} \$ 0,35 \mathrm{~kg}^{-1}$ de feno de Tifton$85, \mathrm{R} \$ 0,55 \mathrm{~kg}^{-1}$ da ração comercial, $\mathrm{R} \$ 0,65 \mathrm{~kg}^{-1}$ de farelo de soja, $\mathrm{R} \$ 0,30 \mathrm{~kg}^{-1}$ de calcário calcítico e $\mathrm{R} \$ 0,80 \mathrm{~kg}^{-1}$ de fosfato bicálcico.

O delineamento experimental utilizado foi o inteiramente casualizado, com cinco tratamentos e cinco repetições, sendo cada cordeiro considerado uma unidade experimental. Os dados foram submetidos à análise de variância e de regressão, com auxílio do pacote estatístico SAS (SAS, 1997). Os modelos foram selecionados com base nos coeficientes de determinação e na significância dos coeficientes de 
regressão, adotando-se o nível de 5\% de probabilidade, utilizando-se o teste F.

\section{RESULTADOS E DISCUSSÃO}

Os valores médios correspondentes a peso vivo inicial, peso vivo ao abate, ganho de peso diário, consumo de matéria seca, conversão alimentar, peso de carcaça quente, rendimento de carcaça quente e aos pesos e porcentagens de quarto, paleta, costilhar, pescoço, vísceras verdes cheias, vísceras verdes vazias e conteúdo gastrintestinal, de acordo com os tratamentos, são apresentados na tabela 2. Verifica-se que o aumento da concentração de volumoso e a diminuição na concentração de concentrado na dieta dos animais promoveram redução linear $(\mathrm{P} \leq 0,01)$ no ganho de peso dos cordeiros, o que proporcionou redução linear $(\mathrm{P} \leq 0,01)$ no peso vivo dos cordeiros no momento do abate. Este aspecto pode ser explicado pela redução da concentração energética das dietas, que ocorreu com o aumento da relação volumoso:concentrado (tabela 1). Os resultados obtidos no presente estudo estão de acordo com CARDOSO et al. (2006), os quais avaliaram o efeito de diferentes níveis de fibra em detergente neutro na dieta de cordeiros confinados na fase de terminação e observaram que o aumento no teor de fibra e de volumoso (silagem de sorgo) promoveu redução linear no ganho de peso diário dos cordeiros, sendo que houve uma redução do ganho de peso em $41,40 \%$ quando se passou do nível de 33,09\% para 75,78\% de volumoso na matéria seca da dieta. Este resultado elevou em 37,3 dias o tempo necessário para que os cordeiros atingissem o peso de abate de $30 \mathrm{~kg}$, estipulado pelos pesquisadores.

Com relação ao consumo de matéria seca, observou-se que o mesmo foi influenciado de forma quadrática pelo aumento no teor de volumoso e pela

Tabela 2 - Valores médios para peso vivo inicial (PVI), peso vivo ao abate (PA), ganho de peso diário total (GMDT), consumo de matéria seca (CMS), conversão alimentar (CA), peso de carcaça quente (PCQ), rendimento de carcaça quente (RCQ) e para os pesos e porcentagens de quarto (QUA), paleta (PAL), costilhar (COST), pescoço (PESC), vísceras verdes cheias (VISCHE), vísceras verdes vazias (VISVAZ) e conteúdo gastrintestinal (CGI), de acordo com os tratamentos.

\begin{tabular}{|c|c|c|c|c|c|c|c|}
\hline & \multicolumn{5}{|c|}{ Relação volumoso:concentrado } & \multirow[b]{2}{*}{ CV (\%) } & \multirow[b]{2}{*}{ ER } \\
\hline & $30: 70$ & $40: 60$ & $50: 50$ & $60: 40$ & $70: 30$ & & \\
\hline PVI (kg) & 15,93 & 17,64 & 17,00 & 17,60 & 16,56 & 20,90 & $Y=16,94$ \\
\hline PA (kg) & 34,13 & 32,60 & 28,82 & 27,02 & 26,08 & 16,56 & 1 \\
\hline GMDT (kg/dia) & 0,228 & 0,187 & 0,148 & 0,123 & 0,119 & 15,63 & 2 \\
\hline CMS (kg/dia) & 0,910 & 0,955 & 0,841 & 0,662 & 0,579 & 5,05 & 3 \\
\hline CA & 4,02 & 5,19 & 5,81 & 5,52 & 5,02 & 16,84 & 4 \\
\hline PCQ (kg) & 14,59 & 14,58 & 12,42 & 10,80 & 10,55 & 16,74 & 5 \\
\hline RCQ (\%) & 42,72 & 44,93 & 43,17 & 39,80 & 40,51 & 5,31 & 6 \\
\hline QUA (kg) & 2,40 & 2,28 & 1,97 & 1,72 & 1,71 & 16,58 & 7 \\
\hline QUA (\%) & 34,24 & 33,23 & 34,72 & 34,47 & 34,53 & 4,18 & $Y=34,16$ \\
\hline PAL (kg) & 1,45 & 1,36 & 1,16 & 1,04 & 1,07 & 18,13 & 8 \\
\hline PAL (\%) & 20,72 & 19,79 & 20,21 & 20,61 & 21,76 & 4,27 & $Y=20,43$ \\
\hline COST (kg) & 2,55 & 2,67 & 2,09 & 1,83 & 1,81 & 20,27 & 9 \\
\hline COST (\%) & 36,39 & 38,66 & 36,36 & 36,10 & 36,56 & 4,68 & $\mathrm{Y}=36,85$ \\
\hline PESC (kg) & 0,62 & 0,58 & 0,53 & 0,44 & 0,35 & 25,65 & 10 \\
\hline PESC (\%) & 8,66 & 8,32 & 9,07 & 8,83 & 7,14 & 12,66 & $\mathrm{Y}=8,38$ \\
\hline VISCHE (kg) & 9,695 & 8,972 & 8,347 & 8,017 & 9,550 & 20,79 & $\mathrm{Y}=8,848$ \\
\hline VISVAZ (kg) & 2,615 & 2,415 & 2,213 & 1,973 & 2,010 & 17,14 & 11 \\
\hline CGI (kg) & 7,080 & 6,557 & 6,134 & 6,044 & 7,540 & 22,67 & $\mathrm{Y}=6,635$ \\
\hline VISCHE (\%) & 28,43 & 27,42 & 28,94 & 29,44 & 36,52 & 10,85 & 12 \\
\hline VISVAZ (\%) & 7,71 & 7,43 & 7,67 & 7,29 & 7,70 & 7,02 & $\mathrm{Y}=7,55$ \\
\hline CGI (\%) & 20,72 & 19,99 & 21,26 & 22,15 & 28,82 & 13,31 & 13 \\
\hline
\end{tabular}

1. $\mathrm{Y}=40,5327-0,2161 * * \mathrm{VOL}, \mathrm{R}^{2}=0,28 ; 2 . \mathrm{Y}=0,2952-0,0027 *$ VOL, $\mathrm{R}^{2}=0,71 ; 3 . \mathrm{Y}=0,80774+0,01090 * \mathrm{VOL}-$ $0,00020757 * * \mathrm{VOL}^{2}, \mathrm{R}^{2}=0,94 ; 4 . \mathrm{Y}=-2,9963+0,3257 * \mathrm{VOL}-0,0030 * \mathrm{VOL}^{2}, \mathrm{R}^{2}=0,30 ; 5 . \mathrm{Y}=18,7572-0,1226 * * \mathrm{VOL}, \mathrm{R}^{2}=0,41 ; 6 . \mathrm{Y}$ $=47,8504-0,1103^{* *}$ VOL, $R^{2}=0,32 ; 7 . \mathrm{Y}=2,9888-0,0194 * * \mathrm{VOL}, \mathrm{R}^{2}=0,41 ; 8 . \mathrm{Y}=1,7428-0,0105^{* *} \mathrm{VOL}, \mathrm{R}^{2}=0,46 ; 9$. $\mathrm{Y}=3,4173$ $-0,0243 * *$ VOL, $\mathrm{R}^{2}=0,38 ; 10 . \mathrm{Y}=0,8516-0,0069 * * \mathrm{VOL}, \mathrm{R}^{2}=0,37 ; 11 . \mathrm{Y}=3,0463-0,0161 * \mathrm{VOL}, \mathrm{R}^{2}=0,26 ; 12 . \mathrm{Y}=19,8374+$ $0,2022 *$ VOL, $R^{2}=0,43 ; 13 . \mathrm{Y}=12,2718+0,2025 * *$ VOL $, \mathrm{R}^{2}=0,47$.

* e ${ }^{* *}$ significativo a 5 e $1 \%$ de probabilidade, respectivamente, pelo teste $\mathrm{F}$.

VOL = nível de volumoso da dieta.

Ciência Rural, v.37, n.5, set-out, 2007. 
redução do teor de concentrado nas dietas, podendose inferir que a ingestão de alimentos foi limitada pelo enchimento para os níveis de 50, 60 e 70\% de volumoso, enquanto que a inclusão de volumoso inferior a $40 \%$ levou à regulação fisiológica do consumo. Este resultado está de acordo com MERTENS (1994) e FORBES (1995), os quais citaram que, quando são fornecidas dietas de alta qualidade, o animal consome para atingir sua demanda energética, sendo este consumo limitado pelo seu potencial genético para utilizar a energia absorvida. Entretanto, quando são fornecidas dietas de baixa qualidade (alto conteúdo de FDN), o consumo de alimento ocorre até atingir o nível de capacidade do trato gastrintestinal. Resultado semelhante ao do presente estudo foi obtido por VÉRAS et al. (2000), os quais, avaliando o consumo de bovinos da raça Nelore, alimentados com rações contendo diferentes níveis de concentrado, verificaram efeito quadrático para o consumo de matéria seca, em kg/dia, sendo o máximo consumo estimado para uma proporção de volumoso de 45,02\%.

Ao avaliar-se a conversão alimentar, observou-se que a mesma foi influenciada de forma quadrática pelas diferentes relações volumoso: concentrado, sendo que a pior conversão foi estimada como 5,84 para uma proporção de 54,3\% de volumoso e $45,7 \%$ de concentrado na dieta. Destaca-se que os cordeiros alimentados com a dieta contendo 30\% de volumoso e $70 \%$ de concentrado apresentaram uma conversão alimentar de 4,02, sendo que este valor concordou com o estudo de CARVALHO et al. (2005), os quais observaram um valor médio de 4,14 para cordeiros confinados e recebendo dieta composta por $60 \%$ de volumoso (feno de Tifton-85) e $40 \%$ de concentrado. O mesmo valor está de acordo também com PILAR et al. (2003), os quais verificaram para cordeiros produtos do cruzamento das raças Ile de France e Merino Australiano, terminados em confinamento com um dieta contendo $20 \%$ de volumoso (Feno de Coast Cross) e 80\% de concentrado, um valor médio para conversão alimentar de 4,02, resultado este idêntico ao do presente estudo.

Quanto ao rendimento de carcaça quente, observa-se que o mesmo diminuiu linearmente $(\mathrm{P} \leq 0,01)$ com o aumento do teor de volumoso nas dietas. Neste sentido, GEAY (1975) afirma que os rendimentos de carcaça com base no peso vivo dos animais são afetados, entre outros fatores, principalmente pelo tempo de jejum e tipo de dieta. Como no presente estudo o tempo de jejum foi semelhante entre os tratamentos, infere-se que houve efeito depressivo causado pelo aumento do teor de volumoso e de FDN na dieta sobre a velocidade de passagem do alimento pelo trato gastrintestinal, o que fez com que houvesse um maior conteúdo digestivo nos cordeiros alimentados com maior proporção de volumoso, no momento do abate. Observou-se também que houve uma redução linear $(\mathrm{P} \leq 0,01)$ no peso de carcaça quente dos animais, sendo esta uma conseqüência na redução do peso vivo ao abate e do rendimento de carcaça verificados. Este resultado pode ser considerado um aspecto negativo do ponto de vista produtivo, pois, quantitativamente, uma menor porção de material comestível resultante do abate estará disponível para o consumidor. Pode-se observar também na tabela 2 que o aumento da relação volumoso:concentrado promoveu efeito linear decrescente $(\mathrm{P} \leq 0,01)$ sobre os pesos de quarto, paleta, costilhar e pescoço, o que pode ser considerado uma conseqüência da redução do peso de carcaça verificado, pois, quando avaliou-se a proporção dos diferentes cortes comerciais em relação à carcaça, não foi verificado efeito significativo $(\mathrm{P}>0,05)$.

Verificou-se que o aumento da relação volumoso:concentrado promoveu redução linear $(\mathrm{P} \leq 0,05)$ no peso de vísceras vazias, o que pode ser explicado pela redução no peso de abate verificado. Observou-se também que a elevação do teor de volumoso e a redução no teor de concentrado nas dietas proporcionaram aumento na proporção de vísceras cheias, o que pode ser explicado pelo aumento linear $(\mathrm{P} \leq 0,01)$ verificado na proporção de conteúdo gastrintestinal (CGI) dos cordeiros no momento do abate, conforme comentado anteriormente.

Na tabela 3, são apresentados os resultados referentes à avaliação econômica da alimentação utilizada na terminação dos cordeiros. A receita bruta em relação ao peso vivo e em relação ao peso de carcaça diminuiu linearmente $(\mathrm{P} \leq 0,01)$ com a elevação do teor de volumoso na dieta, como uma conseqüência da redução do peso vivo ao abate e do peso de carcaça verificados. Contudo, quando descontam-se da receita bruta a despesa total com alimentação dos animais durante o período de confinamento (feno + concentrado), verifica-se que, devido ao aumento do custo de alimentação com a elevação do teor de concentrado nas dietas, quando avaliou-se a lucratividade em relação à venda dos cordeiros vivos, não houve efeito significativo $(\mathrm{P}>0,05)$, tendo sido encontrado um valor médio para lucratividade de $\mathrm{R} \$$ 57,00 animal $^{-1}$. Por outro lado, quando avaliou-se a lucratividade em relação à venda da carcaça dos cordeiros, verificou-se que a mesma diminuiu linearmente $(\mathrm{P} \leq 0,05)$ com o aumento do teor de volumoso na dieta devido à redução do peso de carcaça verificado no abate, o que pode ser considerado um fator limitante da terminação de cordeiros em sistema 
Tabela 3 - Valores médios para consumo de matéria natural (CMN), consumo de matéria natural de feno (CMNFE), consumo de matéria natural de concentrado (CMNCON), despesa diária com feno (DDF), despesa diária com concentrado (DDC), despesa diária total (DDT), despesa total (DT), receita bruta em relação ao peso vivo (RPV), receita bruta em relação às carcaças (RC), lucro em relação ao peso vivo (LPV) e lucro em relação às carcaças (LC), de acordo com os tratamentos.

\begin{tabular}{|c|c|c|c|c|c|c|c|}
\hline & \multicolumn{5}{|c|}{ Relação volumoso:concentrado } & \multirow[b]{2}{*}{ CV (\%) } & \multirow[b]{2}{*}{ ER } \\
\hline & $30: 70$ & $40: 60$ & $50: 50$ & $60: 40$ & 70:30 & & \\
\hline CMN (kg/dia) & 0,954 & 1,051 & 0,928 & 0,725 & 0,632 & 5,97 & 1 \\
\hline CMNFE (kg/dia) & 0,286 & 0,420 & 0,464 & 0,435 & 0,442 & 1,34 & 2 \\
\hline CMNCON (kg/dia) & 0,668 & 0,631 & 0,464 & 0,290 & 0,190 & 7,71 & 3 \\
\hline $\mathrm{DDF}(\mathrm{R} \$ / \mathrm{an} / \mathrm{dia})$ & 0,10 & 0,15 & 0,16 & 0,15 & 0,15 & 5,28 & 4 \\
\hline DDC (R\$/an/dia) & 0,37 & 0,35 & 0,26 & 0,17 & 0,11 & 7,06 & 5 \\
\hline DDT (R\$/an/dia) & 0,47 & 0,50 & 0,42 & 0,32 & 0,27 & 6,29 & 6 \\
\hline $\mathrm{DT}(\mathrm{R} \$ / \mathrm{an})$ & 37,40 & 39,76 & 33,84 & 25,45 & 21,30 & 6,18 & 7 \\
\hline $\mathrm{RPV}$ (R\$/an) & 102,40 & 97,80 & 86,46 & 81,06 & 78,24 & 16,56 & 8 \\
\hline RC (R\$/an) & 138,57 & 138,53 & 117,91 & 102,60 & 100,16 & 16,74 & 9 \\
\hline LPV (R\$/an) & 65,00 & 58,04 & 52,62 & 55,61 & 56,94 & 25,92 & $Y=57,00$ \\
\hline LC (R\$/an) & 101,18 & 98,77 & 84,07 & 77,15 & 78,85 & 22,43 & 10 \\
\hline
\end{tabular}

1. $\mathrm{Y}=0,6770+0,0199 * * \mathrm{VOL}-0,0003 * * \mathrm{VOL}^{2}, \mathrm{R}^{2}=0,91 ; 2 . \mathrm{Y}=-1,4370+0,1025 * * \mathrm{VOL}-0,0018^{* *} \mathrm{VOL}^{2}+0,0001 * * \mathrm{VOL}^{3}, \mathrm{R}^{2}=0,99$ 3. $\mathrm{Y}=1,1224-0,0134 * * \mathrm{VOL}, \mathrm{R}^{2}=0,97 ; 4 . \mathrm{Y}=-0,0916+0,0091 * * \mathrm{VOL}-0,0001 * * \mathrm{VOL}^{2}, \mathrm{R}^{2}=0,84 ; 5 . \mathrm{Y}=0,61589-0,00723 * * \mathrm{VOL}$, $\mathrm{R}^{2}=0,97 ; 6 . \mathrm{Y}=0,43554+0,00541 * \mathrm{VOL}-0,000114 * * \mathrm{VOL}^{2}, \mathrm{R}^{2}=0,93 ; 7 . \mathrm{Y}=32,80042+0,51632 * \mathrm{VOL}-0,00997 * * \mathrm{VOL}^{2}, \mathrm{R}^{2}=0,94$; 8. $\mathrm{Y}=121,5982-0,6485 * *$ VOL, $\mathrm{R}^{2}=0,28 ; 9$. $\mathrm{Y}=178,1668-1,1648 * * \mathrm{VOL}, \mathrm{R}^{2}=0,41 ; 10 . \mathrm{Y}=121,19343-0,66357 * \mathrm{VOL}, \mathrm{R}^{2}=0,19$. $* \mathrm{e} * *$ significativo a 5 e $1 \%$ de probabilidade, respectivamente, pelo teste $\mathrm{F}$.

VOL = nível de volumoso da dieta.

de confinamento. Este resultado confirma a afirmativa de GASTALDI \& SOBRINHO (1998) de que a maximização do uso de alimentos concentrados na terminação de ovinos em confinamento, geralmente, aumenta o custo de produção; entretanto, permite rações com maior concentração de nutrientes, o que pode ser interessante do ponto de vista produtivo.

\section{CONCLUSÃO}

O ganho de peso vivo, o peso de carcaça, os pesos de quarto, paleta, costilhar e pescoço, bem como a lucratividade em relação à venda da carcaça dos cordeiros, diminuem linearmente com a elevação do teor de volumoso e com a redução no teor de concentrado na dieta de cordeiros terminados em confinamento. Além disso, há uma redução linear do rendimento de carcaça devido ao aumento da proporção de conteúdo gastrintestinal no momento do abate dos cordeiros.

\section{AGRADECIMENTOS}

Os autores agradecem à empresa BÜNGE Alimentos, pelo fornecimento do alimento concentrado utilizado; ao Sr. Edmilson Ferreira, pelos animais e infra-estrutura necessários para a condução deste estudo; e ao Centro Universitário Feevale, pelo apoio oferecido.

\section{REFERÊNCIAS}

BENDAHAN, A.B. Confinamento de cordeiros: uma alternativa na ovinocultura. Capturado em 31 jul. 2006. Online. Disponível na Internet http://www.agroline.com.br/ artigos/artigo.php?id=304.

CARDOSO, A.R. et al. Consumo de nutrientes e desempenho de cordeiros alimentados com dietas que contêm diferentes níveis de fibra em detergente neutro. Ciência Rural, v.36, n.1, p.215-221, 2006.

CARVALHO, P.A. Crescimento e composição da carcaça e dos cortes comerciais de cordeiros submetidos à restrição alimentar antes ou após o nascimento. 2005. 198f. Tese (Doutorado em Zootecnia) - Curso de Pós-graduação em Zootecnia, Universidade Federal de Lavras.

CARVALHO, S. et al. Desempenho e características quantitativas da carcaça de cordeiros da raça Suffolk, castrados e não castrados, terminados em confinamento. Revista Brasileira de Agrociência, v.11, n. 1, p. 79-84, 2005.

FORBES, J.M. Voluntary food intake and diet selection in farm animals. Wallington: $\mathrm{CAB}, 1995$. 532p.

GASTALDI, K.A.; SOBRINHO, A.G.S. Desempenho de ovinos F1 Ideal x Ile de France em confinamento com diferentes relações concentrado:volumoso. In: REUNIÃO ANUAL DA SOCIEDADE BRASILEIRA DE ZOOTECNIA, 35., 1998, Botucatu, SP. Anais... Botucatu: Sociedade Brasileira de Zootecnia, 1998. p.257-259.

GEAY, Y. Live wight measurements. In: EEC SEMINAR ON CRITERIAAND METHODS FOR ASSESSMENT OF CARCASS 
AND MEAT CHARACTERISTICS IN BEEF PRODUCTION EXPERIMENTS, 1975, Zeist. Proceedings ... snt, 1975. p.35-42.

MERTENS, D.R. Análise da fibra e sua utilização na avaliação de alimentos e formulação de rações. In: SIMPÓSIO INTERNACIONAL DE RUMINANTES, 1992, Lavras. Anais... Lavras: Sociedade Brasileira de Zootecnia, 1992. p. $188-219$

MERTENS, D.R. Regulation of forage intake. In: FAHEY Jr, G.C. Forage quality, evaluation, and utilization. Madison, WI: Am Soc Agron, 1994. p.450-493.

OSÓRIO, J.C.S. et al. Métodos para avaliação da produção de carne ovina, in vivo, na carcaça e na carne. Pelotas: UFPEL, 1998. 107p.
PILAR, R.C. et al. Desempenho de cordeiros Merino Australiano e cruza Ile de France x Merino Australiano. Ciência e Agrotecnologia, Edição especial, p.1652-1661, 2003.

SAS - Statistical Analysis System. User's guide. Versão 6. SAS INSTITUTE INC. 4.ed. North Caroline, 1997. 846p.

SILVA, D.J. Análise de alimentos (métodos químicos e biológicos). Viçosa: UFV, 1990. 165p.

SIQUEIRA, E.R. et al. Estudo comparativo da recria de cordeiros em confinamento e pastagem. Revista Veterinária e Zootecnia, v.5, p.17-28, 1993.

VÉRAS, A.S.C. et al. Consumo e digestibilidade aparente em bovinos Nelore, não-castrados, alimentados com rações contendo diferentes níveis de concentrado. Revista Brasileira de Zootecnia, v.29, n.6, p.2367-2378, 2000. 